\title{
Efficacy and safety of Afalaza in men with symptomatic benign prostatic hyperplasia at risk of progression: a multicenter, double-blind, placebo-controlled, randomized clinical trial
}

Dmitry Pushkar ${ }^{1}$, Andrey Vinarov², Leonid Spivak², Konstantin Kolontarev ${ }^{1}$, Mikhail Putilovskiy ${ }^{3}$, Elena Andrianova ${ }^{3}$, Oleg Epstein ${ }^{4}$

${ }^{1}$ Moscow State University of Medicine and Dentistry, Department of Urology, Moscow, Russian Federation

${ }^{2}$ Sechenov University, Moscow, Russian Federation

${ }^{3}$ OOONPF Materia Medica Holding Moscow, Russian Federation

${ }^{4}$ The Institute of General Pathology and Pathophysiology, Moscow, Russian Federation

Citation: Pushkar D, Vinarov A, Spivak L, et al. Efficacy and safety of Afalaza in men with symptomatic benign prostatic hyperplasia at risk of progression: a multicenter, double-blind, placebo-controlled, randomized clinical trial. Cent European J Urol. 2018; 71: 427-435.

Article history

Submitted: Oct. 9, 2018

Accepted: Dec. 4, 2018

Published online: Dec. 27, 2018

Corresponding author Konstantin Kolontarev Urology Department of Moscow State University of Medicine and Dentistry 127206 Moscow, Russia 21/2 Vucheticha Str. phone: +7 9169588265 kb80@yandex.ru
Introduction In order to investigate the efficacy and safety of Afalaza in men with benign prostatic hyperplasia (BPH) at risk of progression, this multicenter, double-blind, placebo-controlled, randomized clinical trial was performed. Derived by technological treatment of antibodies to prostate-specific antigen (PSA) and endothelial nitric oxide synthase (eNOs), Afalaza was previously proved to modulate its molecular targets. The mechanism of action of the drug is associated with the modulating effect of the antibiodies (RA-Abs) on the molecular targets (PSA and eNOS) by way of conformational changes.

Material and methods A total of 249 patients aged 45-60 years with BPH and moderate lower urinary tract symptoms (LUTS), total prostate volume (TPV) $\geq 30 \mathrm{~cm}^{3}$, Qmax 10-15 ml/s, and serum PSA<4 ng/ml were randomly assigned to receive either Afalaza $(n=125)$ or placebo $(n=124)$ for 12 months. Changes in BPH/LUTS symptoms (according to the International Prostate Symptom Score), Qmax, TPV, PSA, BPH clinical progression, occurrence of acure urinary retention (AUR) events or BPH-related surgery were estimated as the study endpoints.

Results IPSS mean change was $-3.7 \pm 3.0(95 \% \mathrm{Cl}-4.3$ to -3.2$)$ after 12 months of Afalaza (vs. $-2.9 \pm 2.4 ; 95 \% \mathrm{Cl}$ -3.3 to -2.4 in placebo; $p=0.02$ ). Qmax growth was $2.5 \pm 4.3 \mathrm{ml} / \mathrm{s}$ (vs. $1.4 \pm 3.3$ in placebo; $p=0.049$ ), TPV reduced by $11.8 \pm 16.0 \%$ (vs. $6.5 \pm 14.7 \% ; p=0.01$, and PSA remained unchanged. Afalaza therapy resulted in a significant decrease in the total sum of BPH progression symptoms ( $p=0.01)$. The maximum effect of Afalaza was registered after 12 months without a tendency to form a 'plateau'. During the study, no patients experienced AUR or BPH-related surgery.

Conclusions A 12-month course of Afalaza therapy is effective and safe for patients with BPH. The results of end points measurements revealed asignificant advantage of Afalaza compared to placebo in the overall symptoms benefit and a decline in the risk of BPH progression.

ClinicalTrials.gov: NCT01716104.

Key Words: Afalaza 〈〉 benign prostatic hyperplasia «` BPH progression symptoms «) released-activity

\section{INTRODUCTION}

Benign prostatic hyperplasia (BPH) and associated lower urinary tract symptoms (LUTS) are a prevalent condition in aging men $[1,2]$. Untreated $\mathrm{BPH}$ is complicated by acute urinary retention (AUR), urinary tract infection, renal insufficiency and other disorders [3]. BPH progression hasa 
substantial impact on the social and functional domains of life.

LUTS deterioration is one of the most frequent $\mathrm{BPH}$ progression events. AUR emergence is an urgent symptom related to disease progression $[3,4,5]$. The risk of AUR significantly increases as LUTS severity rises to $>7$ points according to the International Prostate Symptom Score (IPSS) or American Urological Association Symptom Index (AUA-SI) [4, 5]. Prostate volume $>30 \mathrm{~cm}^{3}$, serum prostate-specific antigen (PSA) $\geq 1.4 \mathrm{ng} / \mathrm{ml}$, maximum uroflow rate (Qmax) $<12 \mathrm{ml} / \mathrm{s}$ are known to be risk factors for AUR and the need for BPH related surgery [5]. These factors are considered as $\mathrm{BPH}$ progression symptoms [3, 5, 6, 7].

Preventive treatment that will hamper prostatic hyperplasia and alleviate urinary tract obstruction is a difficult task. The symptoms of emerging BPH are managed by 5 - $\alpha$-reductase inhibitors (5-ARIs), and/or $\alpha$-adrenoblockers (ABs) [8-12]. Type-5 phosphodiesterase (PDE5) inhibitors, antimuscarinics, and $\beta 3$-adrenoreceptor agonists can also be used in treatment $[8,13]$. Prolonged regimens may lead to adverse events (AEs) and complications [8, 14, 15]. For BPH management, Afalaza therapy can be used [16]. It is a medicinal product containing releasedactive forms $[17,18]$ of antibodies (RA-Abs) to prostate-specific antigen (PSA) [19, 20] and endothelial nitric oxide synthase (eNOS) [21, 22]. The pharmacological activity of the medication is associated with the RA-Abs modulating effect on their molecular targets (PSA and eNOS) by way of conformational changes $[17,18]$. RA-Abs to PSA modifies the activity of endogenous PSA [19, 20], enhancing its regulatory effect on metabolic and functional processes in the prostate [23, 24]. RA-Abs to eNOS targets eNOS $[21,22]$ and stimulates production of NO involved in a range of metabolic processes, oxidative stress reactions, maintenance of circulation homeostasis, as well as in the regulation of inflammation processes [25]. The present study was conducted to investigate therapeutic and secondary preventive efficacy and safety of 12-month long Afalaza therapy in men with $\mathrm{BPH}$ at risk of progression.

\section{MATERIAL AND METHODS}

\section{Study overview}

The international, multicenter, double-blind, placebo-controlled, randomized, parallel-group clinical trial was performed between 02/Nov/2012 and 09/Nov/2016 in 15 medical institutions in Russia and in 1 institution in the Ukraine. The study was conducted in accordance with the principles of the
Declaration of Helsinki and Good Clinical Practice and was approved by the institutional review boards. Signed informed consent was obtained from all participants prior to enrollment. In compliance with regulatory requirements (local/national rules) the protocol of the study was submitted and approved by the Ministry of Healthcare of the Russian Federation. The study was registered in the international database ClinicalTrials.gov (ClinicalTrials.gov identifier: NCT01716104).

The study consisted of two phases: 1 - screening for up to 1 week; 2 - treatment and follow-up for 12 months. During the screening period, the patients were examined by an urologist, transrectal ultrasound (TRUS), uroflowmetry, and laboratory tests were performed (Visit 1). Eligible patients were randomized into Group 1 (Afalaza) or Group 2 (placebo) on Visit 2. During the study, the patients paid five more visits to the medical center: after 1, 3, 6, 9, and 12 months. Patient examination, therapy control and compliance assessment were performed at every visit.

\section{Patient selection}

The study included men aged 45-60 years old with $\mathrm{BPH}$ and associated LUTS persisting for more than 3 months, IPSS ranging 8-15 points, total prostate volume (TPV) $>30 \mathrm{~cm}^{3}$, Qmax varying $10-15 \mathrm{ml} / \mathrm{s}$; voided volume between 125 and $350 \mathrm{ml}$; post-void residual urine volume $<100 \mathrm{ml}$, and serum PSA $<4 \mathrm{ng} / \mathrm{ml}$. The inclusion criteria were very precise and restricted in order to prove the efficacy of Afalaza monotherapy in prevention of disease progression, to provide comparability of the two groups, to mitigate risks for patients on placebo-therapy during 12-month participation in the clinical trials, and guarantee non-exclusion for them.

The exclusion criteria were a history of invasive or surgical treatment for $\mathrm{BPH}$, AUR in the previous 3 months, suspected prostate cancer, urolithiasis, neurogenic lower urinary tract dysfunction, bladder diverticulum, urinary tract infection, urethral stricture, bladder neck sclerosis, severe concomitant pathology, renal or hepatic failure, history of 5-ARIs use.

\section{Patient assessment}

In the screening period (one week) the patients were examined to prove that BPH (not any other condition) caused lower urinary tract symptoms (LUTS) including bladder outlet obstruction (BOO) symptoms.

IPSS questionnaire and Qmax were carried out at screening, after 1, 3, 6, and 12 months; TRUS 
for TPV measurement was implemented at screening and every 3 months; serum PSA was measured at screening, after 6, and 12 months; hematology, blood chemistry, and urinalysis were checked at screening and after 12 months.

Moderate-to-severe symptoms (according to IPSS), depressed Qmax rate $(<12 \mathrm{ml} / \mathrm{s})$, enlarged prostate $\left(>30 \mathrm{~cm}^{3}\right)$ or elevated serum PSA $(\geq 1.4 \mathrm{ng} / \mathrm{ml})$ were considered as risk factors for BPH progression [4-7]. Each particular risk factor scored 1 point, its absence 0 point, thus maximum score equaled 4 points. Each risk factor change was estimated after 12 months. Increase in risk factor severity (symptom deterioration) in comparison with initial value added 1 point, lack of changes gave 0 point, and decrease (symptom benefit) meant minus 1 point. The total sum dynamics was used to make a conclusion about $\mathrm{BPH}$ progression (in case of the score increase) or retardation (in case of the score decrease).

Laboratory tests and AEs registration were performed to assess the safety of the treatment. AEs were categorized by organ system, preferable terms, severity, and treatment relatedness as determined by the urologist.

\section{Randomization and blinding}

After screening, the patients were assigned to Afalaza or placebo groups in a 1:1 ratio. Interactive voice/web response randomization system based on a random number generator was used for patients randomization and investigational medicine assignment. Neither participants, nor investigators, trial centers staff, and the sponsor's project team were aware of the treatment group assignment throughout the duration of the study.

Manufacturing, packaging and labeling with unique identification codes of the double-blind medications (Afalaza or placebo/identical in shape and taste tablet containing excipients) were performed by $\mathrm{OOO}$ 'NPF' 'Materia Medica Holding'.

\section{Treatment}

Two Afalaza tablets were administered twice a day, 15 to 30 minutes before meals for 12 months; the tablets were kept in the mouth until completely dissolved. Each Afalaza tablet contains a mixture of affinity purified polyclonal antibodies to PSA $(6 \mathrm{mg})$ and antibodies to eNOS $(6 \mathrm{mg})$ in released-active form produced by the patented technology (US Patent $8,535,664 \mathrm{~B} 2,2013)$ in accordance with the applicable European Pharmacopeia requirements.

Placebo was administered according to Afalaza administration schedule.
The compliance with the study therapies was assessed at each visit according to the count of tablets returned by the patient.

The patients were allowed to use concomitant therapy excluding 5-ARIs, ABs, herbal extracts and other drugs for the treatment of $\mathrm{BPH}$ and urination disorders; testosterone hormones, PDE5 inhibitors, diuretics, neuroleptics, psychoanaleptics, nebivolol, long-acting nitrates. These medications were not permitted either within 3 months prior to study entry, and 5-ARIs were prohibited at any point previously.

\section{Study end points and statistical analysis}

The primary efficacy end point was BPH symptom alleviation in IPSS score at 1, 3, 6 and 12 months compared to the baseline. Changes in the Qmax, TPV, PSA, BPH-related health status (question 8 in the IPSS), and dynamics of the total sum of BPH progression symptoms were used as the secondary end points. AUR episodes and/or BPH related surgery were monitored during the study.

Statistical analysis was performed using SAS (Version 9.4) statistical software. Data of the full analysis set excluding the failure to satisfy major entry criteria (eligibility violations) were used for intention-to-treat (ITT) analysis. The sample size was calculated assuming the difference between changes in the average IPSS score after 12 months compared to the baseline in Afalaza group and in Placebo group would be 1.0 point; while the standard deviation (SD) would be 2.5 points. The power of statistical criteria was assumed to be $80 \%$; the type I error was assumed to be $<5 \%$. The minimum required size for each group was 100 patients; at least 260 patients had to be included, taking into account the dropout rate (during the screening and the study period) set at $1.2(20 \%)$ or greater.

To compare the results in two groups, Student's t-test was used for continuous variables, and the Holm adaptive method was used for multiple comparisons. The longitudinal data was analyzed with two-way analysis of covariance (ANCOVA; with the factors based on 2 levels for the Group and 4 levels for the Visit, and adjustment for covariates for Visit 1) using SAS PROC MIXED. The $\chi^{2}$ criterion, Cochran-Mantel-Haenszel test, and Fisher's exact test were used to compare the proportions.

\section{RESULTS}

Patient demographics and baseline characteristics

A total of 260 patients were assessed for eligibility. Upon completion of the study screening, 11 patients 
were excluded as they did not meet the inclusion criteria or did meet the exclusion criteria. In total, 249 patients were randomized to treatment: 125 of them into the Afalaza group and 124 into the Placebo group (Figure 1). Thirteen randomized patients (6 in Afalaza group and 7 in Placebo group) failed to satisfy major entry criteria (eligibility violations), so they were excluded from the full analysis set. The rest of the participants $(n=236)$ constituted the ITT set analyzed. Treatment outcomes in this set (119 patients in Afalaza group and 117 in Placebo group) were considered for ITT analysis of efficacy. Treatment safety in both groups was assessed in all patients who received at least one dose of Afalaza $(\mathrm{n}=125)$ or placebo $(\mathrm{n}=124)$.

Patients in both groups did not differ in demographic and baseline characteristics, including IPSS score, Qmax depression, prostate volume enlargement, PSA level, and total sum of the risk factors for $\mathrm{BPH}$ progression (Table 1).

\section{Change in International Prostate Symptom \\ Score}

After 12 months, the IPSS mean decreased to 8.1 \pm 3.0 (95\% confidence interval [CI] 7.5 to 8.6 ) in patients of the Afalaza group versus $8.6 \pm 2.5$ (95\% CI 8.1 to 9.0 ) in the Placebo group. ANCOVA revealed significant results for the Group factor $(p=0.001)$, for the Visit factor $(p<0.0001)$, and for the Groupby-Visit interaction $(p=0.008)$. Thus, ANCOVA confirmed that IPSS decrease was more significant in the Afalaza group than in the Placebo group.

Final IPSS score decline from baseline in the Afalaza group was $-3.7 \pm 3.0$ versus $-2.9 \pm 2.4$ in the Placebo group (Figure 2). The Student's t-test has proved a significant IPSS decrease at each visit $(1,3,6$, and 12 months of treatment) for Afalaza patients compared to that of the Placebo group.

\section{Maximum urinary flow rate}

At 1 month follow-up, Qmax mean increase was higher for the Afalaza group in comparison to the Placebo group (Figure 3). Subsequent administration of Afalaza led to Qmax rise to $14.9 \pm 4.3 \mathrm{ml} / \mathrm{s}$ versus 14.0 $\pm 3.2 \mathrm{ml} / \mathrm{s}$ in the Placebo group at 12 months followup. ANCOVA proved Qmax increase to be more significant in the Afalaza group than in the Placebo group (Group factor: $p=0.0004$; Visit factor: $p<0.0001$; Group-by-Visit interaction: $\mathrm{p}=0.0335$ ). Total Qmax change confirmed its significant growing by the end of the 12 months for the Afalaza group, amounting to $+2.5 \pm 4.3 \mathrm{ml} / \mathrm{s}$ (vs. $+1.4 \pm 3.3 \mathrm{ml} / \mathrm{sec}$ in Placebo group; $\mathrm{p}=0.049$ ).

\section{Total prostate volume}

In the Afalaza group, mean TPV decreased by 3.0 $\pm 6.3 \mathrm{~cm}^{3}$ after 3 months, by $4.5 \pm 6.6 \mathrm{~cm}^{3}$ after

Table 1. Patient demographics and baseline characteristics

\begin{tabular}{|c|c|c|c|}
\hline & \multicolumn{2}{|c|}{ Group } & \multirow[b]{2}{*}{ p-value } \\
\hline & $\begin{array}{l}\text { Afalaza } \\
(n=119)\end{array}$ & $\begin{array}{l}\text { Placebo } \\
(\mathrm{n}=117)\end{array}$ & \\
\hline \multicolumn{4}{|l|}{ Age (years) } \\
\hline Median & 55.1 & 54.8 & $0.90^{*}$ \\
\hline Minimum & 45.0 & 45.4 & \\
\hline Maximum & 60.9 & 60.9 & \\
\hline IPSS (M $\pm S D)$, points & $11.8 \pm 2.0$ & $11.4 \pm 1.8$ & 0.16 \\
\hline $\operatorname{Qmax}(\mathrm{M} \pm \mathrm{SD}), \mathrm{ml} / \mathrm{s}$ & $12.4 \pm 1.5$ & $12.6 \pm 1.4$ & 0.48 \\
\hline Prostate volume $(\mathrm{M} \pm \mathrm{SD}), \mathrm{cm}^{3}$ & $43.1 \pm 17.9$ & $40.5 \pm 10.1$ & 0.17 \\
\hline $\mathrm{PSA}(\mathrm{M} \pm \mathrm{SD}), \mathrm{ng} / \mathrm{ml}$ & $1.5 \pm 1.0$ & $1.5 \pm 0.9$ & 0.98 \\
\hline $\begin{array}{l}\mathrm{BPH} \text {-related health status } \\
(\mathrm{M} \pm \mathrm{SD}) \text {, points }\end{array}$ & $3.3 \pm 0.9$ & $3.3 \pm 1.0$ & 0.87 \\
\hline $\begin{array}{l}\text { Total sum of the risk factors } \\
\text { for BPH progression }(\mathrm{M} \pm \mathrm{SD}) \text {, } \\
\text { points }\end{array}$ & $2.8 \pm 0.7$ & $2.8 \pm 0.7$ & 0.61 \\
\hline $\begin{array}{l}\text { Number of patients with } \\
\text { at least one concomitant } \\
\text { disease }(n / \%)\end{array}$ & $52 / 43.7$ & $48 / 41.0$ & $0.70 * *$ \\
\hline $\begin{array}{l}\text { Number of patients received } \\
\text { at least one concomitant } \\
\text { medication }(\mathrm{n} / \%)\end{array}$ & $35 / 29.4$ & $33 / 28.2$ & $0.88 * *$ \\
\hline
\end{tabular}

${ }^{*} \chi^{2}$ Median One-Way Analysis. IPSS, $\mathrm{Q}_{\text {max }}$ Prostate volume, PSA, BPH-related health status, and Total sum of the risk factors for BPH progression were analyzed by Student's t-test

**Fisher's exact test.IPSS - International Prostate Symptom Score;

$\mathrm{Q}_{\max }$ - maximum uroflow rate. PSA - prostate-specific antigen; $\mathrm{BPH}$ - benign prostatic hyperplasia

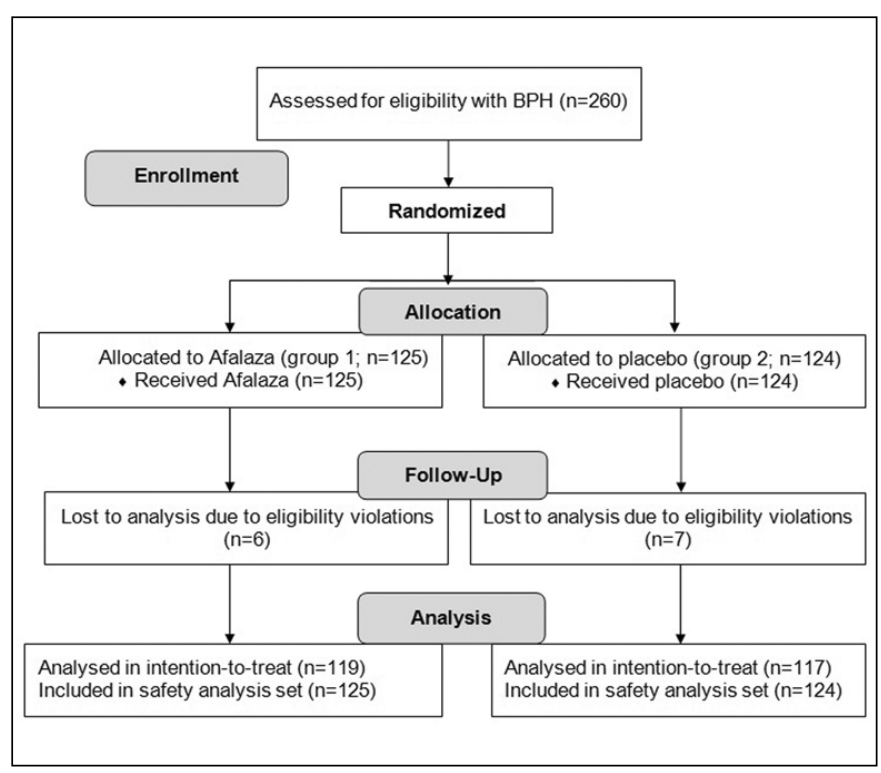

Figure 1. Study design flow diagram. 


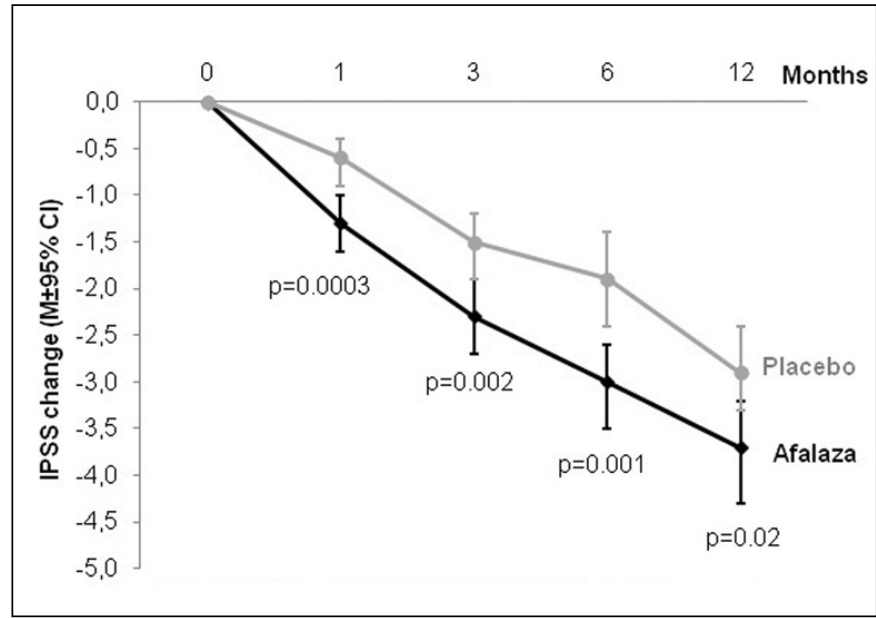

Figure 2. Mean changes in International Prostate Sypmtom Score (IPSS) score during 12 months.

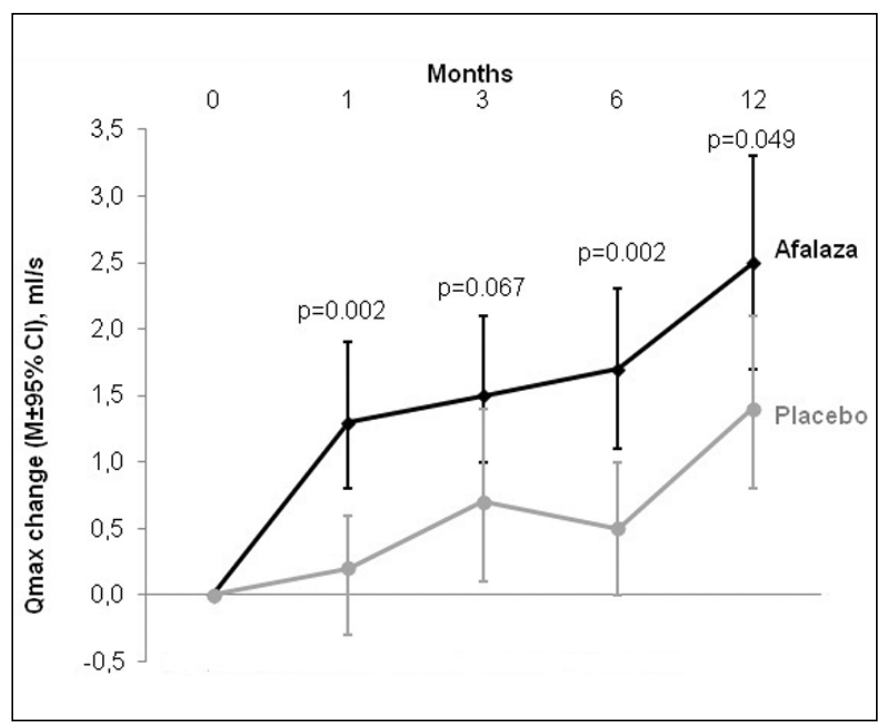

Figure 3. Mean changes in Qmax during 12 months.

6 months, and by $5.5 \pm 8.0 \mathrm{~cm}^{3}$ after 12 months. In the Placebo group, as measured at the same time intervals, the mean TPV changes were $-1.2 \pm 4.8$ $(\mathrm{p}=0.0195),-1.9 \pm 5.5(\mathrm{p}=0.0019)$, and $-2.5 \pm 5.7$ $(\mathrm{p}=0.0018) \mathrm{cm}^{3}$. Scored as the percentage of the initial volume, these values are presented in Figure 4. Student's t-test showed superiority for Afalaza over placebo in steady TPV reduction both in $\mathrm{cm}^{3}$ and percentage units.

\section{Prostate-specific antigen}

The mean values of total PSA remained stable throughout the study in both groups (Table 2). ANCOVA proved that mean PSA did not differ between the two groups during the 12 months of treatment.

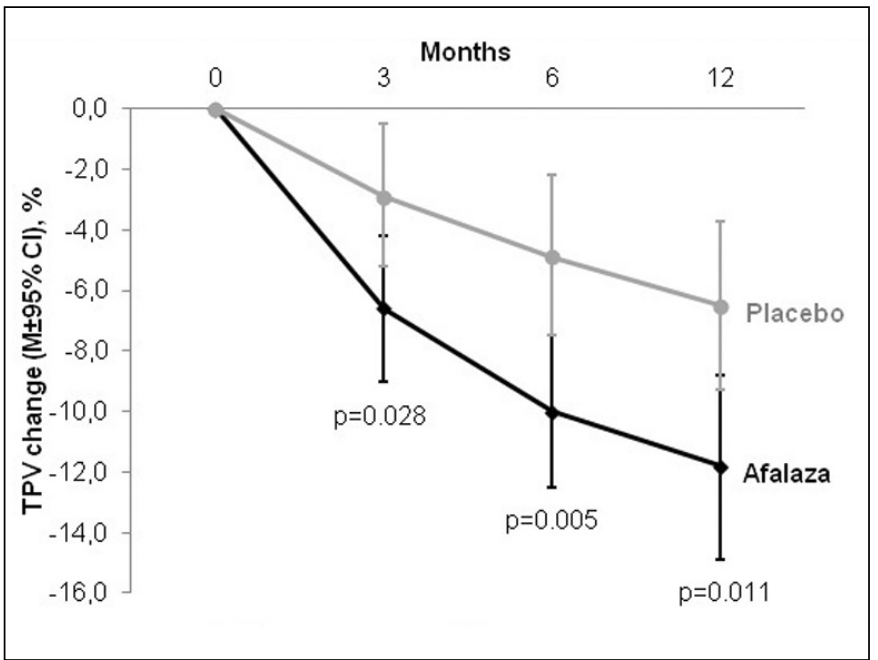

Figure 4. Mean changes in total prostate volume (\%) during 12 months.

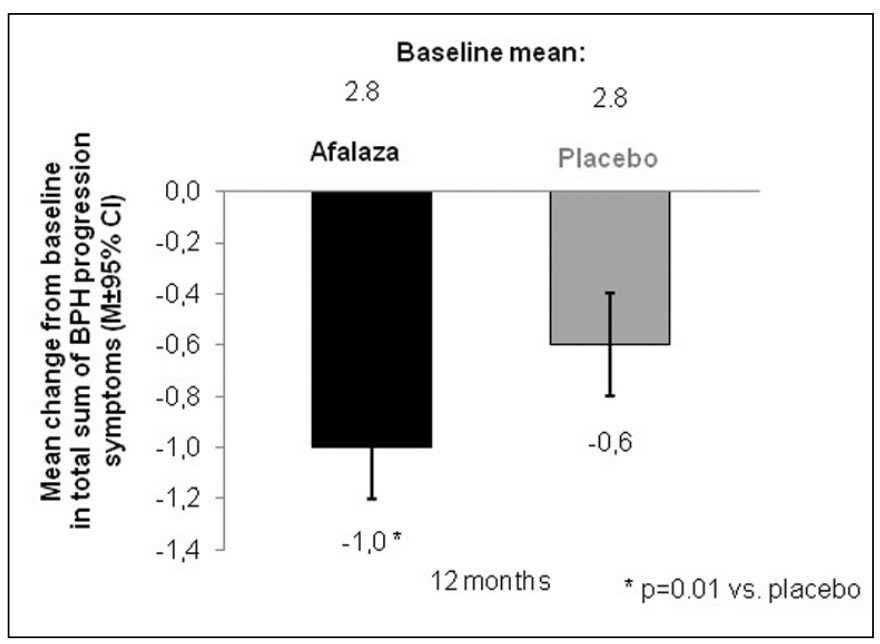

Figure 5. Mean changes in total sum of the risk factors for $\mathrm{BPH}$ progression during 12 months.

PSA increase was reported in 9 patients: 4 patients in the Afalaza group and 5 patients in the Placebo group ( $\mathrm{p}=0.54)$. All 9 patients fully completed their participation in the study and received further treatment.

\section{Benign prostatic hyperplasia-related health status}

In the Afalaza group, the IPSS question 8 score decreased from the baseline $(-1.0 \pm 1.0 ; 95 \%$ CI $-1.2 \div-0.8)$ and differed favorably and significantly from the Placebo group $(-0.6 \pm 1.2 ; 95 \%$ CI $-0.9 \div-0.4 ; \mathrm{p}=0.025)$. ANCOVA results confirmed the advantage of Afalaza over placebo in improving BPH-related health status throughout the 12 months of treatment (Group-byVisit interaction: $\mathrm{p}=0.04$ ). 
Table 2. Serum prostate-specific antigena $(\mathrm{ng} / \mathrm{ml})$

\begin{tabular}{|c|c|c|c|c|}
\hline \multirow{2}{*}{ Visit/Month } & \multirow{2}{*}{$\begin{array}{l}\text { Afalaza } \\
N=103\end{array}$} & \multirow{2}{*}{$\begin{array}{l}\text { Placebo } \\
\mathrm{N}=105\end{array}$} & \multicolumn{2}{|c|}{ Statistics } \\
\hline & & & Student's t-test ${ }^{b}$ & ANCOVA \\
\hline 1/Baseline & $\begin{array}{c}1.5 \pm 1.0 \\
(1.3 \div 1.7)\end{array}$ & $\begin{array}{c}1.5 \pm 0.9 \\
(1.3 \div 1.7)\end{array}$ & $\begin{array}{l}t=0.03 \\
p=0.98\end{array}$ & $\begin{array}{c}\text { 1) Group factor } \\
F=0.16 ; p=0.69\end{array}$ \\
\hline 5/6 months & $\begin{array}{c}1.6 \pm 1.1 \\
(1.4 \div 1.8)\end{array}$ & $\begin{array}{c}1.7 \pm 1,3 \\
(1.4 \div 1.9)\end{array}$ & $\begin{array}{l}t=-0.70 \\
p=0.48\end{array}$ & $\begin{array}{l}\text { 2) Visit factor: } \\
F=2.4 ; p=0.09\end{array}$ \\
\hline $7 / 12$ months & $\begin{array}{c}1.5 \pm 1,2 \\
(1.2 \div 1.7)\end{array}$ & $\begin{array}{c}1.6 \pm 1,2 \\
(1.4 \div 1.8)\end{array}$ & $\begin{array}{l}t=-0.44 \\
p=0.66\end{array}$ & $\begin{array}{l}\text { 3) Group*bVisit: } \\
F=0.09 ; p=0.91\end{array}$ \\
\hline
\end{tabular}

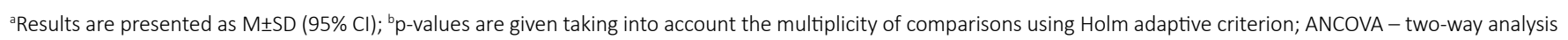
of covariance with adjustment for covariate for Visit 1

Table 3. Adverse events

\begin{tabular}{|c|c|c|c|}
\hline SOC / Adverse event & Afalaza $(\mathrm{N}=125)$ & Placebo $(N=124)$ & $\mathrm{p}$-value \\
\hline Number of subjects reporting at least one $A E^{*}$ & $25(20.0)$ & $29(23.4)$ & 0.54 \\
\hline \multicolumn{4}{|l|}{ Gastrointestinal disorders } \\
\hline At least one event & $4(3.2)$ & $5(4.0)$ & 0.75 \\
\hline Upper abdominal pain & $1(0.8)$ & $0(0.0)$ & 1.00 \\
\hline Inguinal hernia & $1(0.8)$ & $0(0.0)$ & 1.00 \\
\hline Diarrhea & $1(0.8)$ & $0(0.0)$ & 1.00 \\
\hline Heartburn & $0(0.0)$ & $1(0.8)$ & 0.50 \\
\hline Swollen tongue & $0(0.0)$ & $1(0.8)$ & 0.50 \\
\hline Nausea & $1(0.8)$ & $2(1.6)$ & 0.62 \\
\hline Exacerbation of chronic gastritis & $0(0.0)$ & $1(0.8)$ & 0.50 \\
\hline \multicolumn{4}{|l|}{ General disorders } \\
\hline At least one event & $0(0.0)$ & $1(0.8)$ & 0.50 \\
\hline Asthenia & $0(0.0)$ & $1(0.8)$ & 0.50 \\
\hline \multicolumn{4}{|l|}{ Infections and infestations } \\
\hline At least one event & $13(10.4)$ & $13(10.5)$ & 1.000 \\
\hline Gastroenteritis & $0(0.0)$ & $1(0.8)$ & 0.50 \\
\hline Urinary tract infection & $1(0.8)$ & $0(0.0)$ & 1.00 \\
\hline Herpes labialis & $1(0.8)$ & $0(0.0)$ & 1.00 \\
\hline Upper respiratory tract infection & $9(7.1)$ & $10(8.0)$ & 0.82 \\
\hline Acute bronchitis & $1(0.8)$ & $0(0.0)$ & 1.00 \\
\hline Rhinitis & $0(0.0)$ & $2(1.6)$ & 0.25 \\
\hline Pharyngitis & $1(0.8)$ & $0(0.0)$ & 1.00 \\
\hline \multicolumn{4}{|l|}{ Investigations } \\
\hline At least one event & $4(3.2)$ & $4(3.2)$ & 0.54 \\
\hline Blood pressure increased & $0(0.0)$ & $0(0.0)$ & 0.50 \\
\hline Free prostate-specific antigen increased & $4(3.2)$ & $4(3.2)$ & 0.54 \\
\hline \multicolumn{4}{|l|}{ Musculoskeletal and connective tissue disorders } \\
\hline At least one event & $0(0.0)$ & $1(0.8)$ & 0.50 \\
\hline Chronic injury of the medial meniscus & $0(0.0)$ & $1(0.8)$ & 0.50 \\
\hline \multicolumn{4}{|l|}{ Nervous system disorders } \\
\hline At least one event & $1(0.8)$ & $5 *(4.0)$ & 0.12 \\
\hline Headache & $1(0.8)$ & $6(4.8)$ & 0.07 \\
\hline \multicolumn{4}{|l|}{ Respiratory, thoracic and mediastinal disorders } \\
\hline At least one event & $3(2.4)$ & $0(0.0)$ & 0.25 \\
\hline Vasomotor rhinitis & $1(0.8)$ & $0(0.0)$ & 1.00 \\
\hline Throat irritation & $1(0.8)$ & $0(0.0)$ & 1.00 \\
\hline Cough & $1(0.8)$ & $0(0.0)$ & 1.00 \\
\hline \multicolumn{4}{|l|}{ Skin and subcutaneous tissue disorders } \\
\hline At least one event & $0(0.0)$ & $1(0.8)$ & 0.50 \\
\hline Contact dermatitis & $0(0.0)$ & $1(0.8)$ & 0.50 \\
\hline \multicolumn{4}{|l|}{ Vascular disorders } \\
\hline At least one event & $3(2.4)$ & $1(0.8)$ & 0.62 \\
\hline Hypertensive crisis & $2(1.6)$ & $1(0.8)$ & 0.54 \\
\hline Haemorrhoids & $1(0.8)$ & $0(0.0)$ & 1.00 \\
\hline
\end{tabular}

The results are presented as $\mathrm{n}(\%)$. AE - adverse event; SOC - system-organ-class in accordance with Medical Dictionary for Regulatory Activities (MedDRA). ${ }^{*}$ Differences in the number of patients with at least one AE and the total number of AEs in the group are due to the fact that in some patients there were 2,3 or $4 \mathrm{AE}$ registered. ${ }^{* *} \mathrm{In} 1$ patient from the Placebo group PSA was increased twice (after 6 and 12 months of therapy), but the investigator recorded this as 1 AE. 
Total sum of risk factors for benign prostatic hyperplasia progression

In patients of the Afalaza group, the total sum of risk factors for BPH progression was reduced by $-1.0 \pm 1.0$ (95\% CI -1.1 to -0.8) points after 12 months of treatment (Figure 5), which was considerably higher $(\mathrm{p}=0.01)$ than in the Placebo group $(-0.6 \pm 1.0 ; 95 \%$ CI -0.8 to -0.4 ).

\section{Safety and tolerability}

A total of 28 AEs in $25(20.0 \%)$ patients of the Afalaza group and 35 AEs in $29(23.4 \%)$ patients of the Placebo group were reported during the 12 months of treatment, without significant differences between the frequency of AEs related to systems-organ-classes (Table 3).

Twenty-one (75.0\%) AEs in the Afalaza group were unrelated to the medication, in $1(3.6 \%)$ the relationship was uncertain, and in $6(21.4 \%)$ it was possible or probable including PSA increase $(4$ cases vs. 6 cases in placebo, $\mathrm{p}=0.54)$, abdominal pain $(\mathrm{n}=1)$, and herpes labialis $(\mathrm{n}=1)$. In the Placebo group, relationship was absent in $26(74.3 \%)$, uncertain in $4(11.4 \%)$, possible or probable in $5(14.3 \%)$ cases. A total of $75.0 \%$ AEs were mild and $25.0 \%$ AEs were moderate. The frequency of AEs relationship and severity did not differ in both groups (Tables 4,5 ).

Hematology, blood chemistry, and urinalysis parameters exceeded the reference values neither at the enrollment nor at the end of the treatment.

Table 4. Relationship between the drug and adverse events

\begin{tabular}{lccc}
\hline Relationship & $\begin{array}{c}\text { Afalaza } \\
(\mathrm{N}=125)\end{array}$ & $\begin{array}{c}\text { Placebo } \\
(\mathrm{N}=124)\end{array}$ & Statistics \\
\hline Unrelated & $21(75.0)$ & $26(74.3)$ & \\
\hdashline Uncertain & $1(3.6)$ & $4(11.4)$ & \\
\hdashline Possible & $3(10.7)$ & $1(2.9)$ \\
\hdashline Probable & $3(10.7)$ & $4(11.4)$ \\
\hdashline Total & $28(100.0)$ & $35(100.0)$ \\
\hline
\end{tabular}

The results are presented as $n(\%)$. $\mathrm{CMH} \chi^{2}-$ Cochran-Mantel-Haenszel $\chi^{2}$ (modified for multiple comparisons)

Table 5. Adverse events severity

\begin{tabular}{lccc}
\hline \multicolumn{1}{c}{ Severity } & $\begin{array}{c}\text { Afalaza } \\
(\mathrm{N}=125)\end{array}$ & $\begin{array}{c}\text { Placebo } \\
(\mathrm{N}=124)\end{array}$ & Statistics \\
\hline Mild & $21(75.0)$ & $26(74.3)$ & \\
\hdashline Moderate & $7(25.0)$ & $9(25.7)$
\end{tabular}$\quad \chi^{2}=0.004 ; \mathrm{p}=0.95 \mathbf{\nabla}$

The results are presented as $n(\%)$. $\mathbf{\nabla}$ The results of frequency analysis ( $\chi^{2}$ test).
The mean compliance rates in patients of Afalaza and Placebo groups were close to $100 \%$ without significant differences $(\mathrm{p}=0.65)$.

There was no evidence of drug-to-drug interaction with medications administered concomitantly with Afalaza to treat comorbidities in BPH patients.

During the study, no cases of AUR or BPH-related surgery were reported.

\section{DISCUSSION}

The 12-month Afalaza therapy improved LUTS, led to Qmax increase and TPV reduction in men with symptomatic BPH. The therapy effect manifested itself during the first three months, positive dynamics continued throughout the subsequent months and sustained until the end of 12 months. IPSS, Qmax and TPV improvement reached their maximum at the end of the therapy but did not reach a plateau. Serum PSA remained stable throughout the study. To assess the risk of $\mathrm{BPH}$ progression, the proven risk factors were considered, comprising total IPSS, Qmax, TPV, and PSA level [3-7]. Afalaza therapy led to a significant reduction in total sum of $\mathrm{BPH}$ progression symptoms compared to placebo $(\mathrm{p}=0.01)$. For 12 months of therapy none of the participants experienced AUR or conditions requiring surgery.

It may seem challenging to compare the results of this Afalaza study to any other long-term trials aimed to assess the effects of prolonged therapy on $\mathrm{BPH}$-related symptoms and disease progression due to the presence of 5-ARIs and $\mathrm{ABs}$ as a common therapy practice [8-12]. In comparison, finasteride treatment in the 4-year PLESS study improved symptom score by 2.6 points (vs. 1.0 for placebo according to AUA-SI; p <0.001) [9]. The MTOPS study [11] showed that finasteride reduced the risk of symptomatic progression (defined as an increase in the AUA-SI $\geq 4$ points) by $30 \%$ compared with placebo $(\mathrm{p}=0.016)$.

5-ARIs exert an antiandrogenic effect by inhibiting the conversion of testosterone to dihydrotestosterone which leads to a decreased protein synthesis and prostate tissue involution. Afalaza has a completely different mechanism of action. Pharmacological activity of the medicine is associated with modulating effects $[17,18]$ on the PSA and eNOS via modification of its conformation. PSA is shown to stimulate reactive oxygen species generation in $\mathrm{LNCaP}, \mathrm{PC} 3$ and DU145 cells [26]. PSA suppresses the growth and migration of endothelial cells by inhibiting endothelial cell response to the fibroblast growth factor 2 and vascular endothelial growth factor. They have the ability to cleave plasminogen, causing the release of biologically active angiostatin-like fragments and 
can essentially slow the progression of the disease through this antiangiogenic activity [23]. eNOS is known to keep blood vessels dilated, control blood pressure, and have numerous other vasoprotective effects [25]. RA-Abs to PSA and eNOS synergistic action is assumed to result in prostate metabolism, circulation, and proliferation balance leading to the improvement of the urinary tract function. These effects were demonstrated in vivo and in clinical trials for each particular component of Afalaza: RA-Abs to PSA (active substance of Afalaza) [27, 28] and RAAbs to eNOS (active substance of Impaza) [29, 30]. The comparison of the study results with data of international multicenter clinical trials examining the efficacy of the combined (5-ARI $+\mathrm{AB})$ or monocomponent (5-ARI /AB) [7, 9-12] treatment in $\mathrm{BPH}$, highlights a positive benefit-risk balance of Afalaza therapy. The effective and safe effect that Afalaza exerts on the hyperplastic prostate is realized in prostate volume reduction and $\mathrm{BPH}$ symptoms relief. A gradual and augmenting activity of Afalaza achieved the maximum effect after 12 months. $\mathrm{Cu}-$ mulative $\mathrm{BPH}$ symptoms benefit, prostate volume reduction, Qmax rising without reaching a plateau within 12 months indicate that more prolonged Afalaza use may hamper BPH progression.

The positive therapeutic results of Afalaza were combined with a high level of safety. Afalaza neither increased, nor decreased serum PSA concentration. The simultaneous use of Afalaza with drugs of various classes did not lead to incompatibility reactions. Despite more moderate benefit compared to the routinely prescribed 5ARIs and/or ABs the advantage of Afalaza is obvious due to rare frequency of AEs related to the medicine.

The absence of patients with severe LUTS, huge prostate enlargement, severe concomitant diseases, and elderly age may be considered as study limitations. Its relatively short duration and small samples size did not allow to investigate further outcomes of Afalaza monotherapy. In addition, the effects of various Afalaza combinations with drugs for the treatment of $\mathrm{BPH}$ and urination disorders have not been evaluated.

\section{CONCLUSIONS}

The data from this 12-month study in men with moderate LUTS/BPH at risk of progression suggests sustainable symptoms benefits of Afalaza use. They are reflected in the IPSS and BPH-related health status improvement, Qmax growth, possible decline in the prostate enlargement, and alleviation of the total sum of the risk factors for $\mathrm{BPH}$ progression. The conclusions were based on the proved superiority over placebo. Afalaza provides a unique balance of efficacy and safety which favorably distinguishes this drug among others used to treat LUTS/BPH. The total sum of $\mathrm{BPH}$ progression symptoms decrease indicates the possibility of disease control achieving with long-term Afalaza therapy. This assumption requires confirmation in the additional studies in large populations using validated methods for assessing the function and structure of the prostate and urinary tract. To compare the efficacy and safety of Afalaza with standard medication is the next phase of the clinical development program.

\section{CONFLICTS OF INTEREST}

This study was funded by a grant from OOO NPF Materia Medica Holding (9, 3rd Samotyochny per., 127473, Moscow, Russia). Statistical analysis was provided by OOO NPF Materia Medica Holding. Conflict of interest statement Dmitriy Pushkar, Andrey Vinarov, and Leonid Spivak received the investigator's grant from OOO NPF Materia Medica Holding to conduct clinical trial. Mikhail Putilovskiy and Elena Andrianova are employees of the OOO NPF Materia Medica Holding. Oleg Epstein is a founder of the OOO NPF Materia Medica Holding. Afalaza is a drug manufactured by OOO NPF Materia Medica Holding.

\section{ACKNOWLEDGMENTS}

This study was funded by a grant from OOO NPF Materia Medica Holding (9, $3^{\text {rd }}$ Samotyochny per., 127473, Moscow, Russia). Statistical analysis was provided by OOO NPF Materia Medica Holding. The authors thank the research staff at the participating sites. The investigators involved were Igor Aboyan (Municipal Clinical Diagnostic Center Zdorovie, Rostov-on-Don), Mikhail Andryukhin (Peoples' Friendship University of Russia, Moscow), Konstantin Chibirov (Research Institute of Phthisiopulmonology, Saint-Petersburg), Mikhail Evdokimov (Limited Liability Company Family Policlinic No. 4, Korolev), Vadim Surikov (Polyclinic No. 3, Moscow), Andrey Gorelov (Pokrovsky City Hospital, Saint-Petersburg), Sergey Gorelov (Clinical Hospital No. 122, Saint-Petersburg), Vladimir Kheifets (Limited Liability Company Hospital OrCli, Saint-Petersburg), Alexey Kornev (Hospital for veterans of wars No. 2, Moscow), Ekaterina Korshunova (Scientific and Practical Psychoneurological Center, Moscow), Viktor Latypov (Siberian State Medical University, Tomsk), Sergey Petrov (The Nikiforov Russian Center of Emergency and Radiation Medicine, Saint-Petersburg), Alexey Vasilevskiy (Limited Liability Company Medical sanitary unit No. 157, Saint-Petersburg), and Igor Gorpinchenko (Institute of Urology, Kiev, Ukraine). 


\section{References}

1. Egan KB. The Epidemiology of Benign Prostatic Hyperplasia Associated with Lower Urinary Tract Symptoms: Prevalence and Incident Rates. Urol Clin North Am. 2016; 43: 289-297.

2. Speakman M, Kirby R, Doyle S, Ioannou C. Burden of male lower urinary tract symptoms (LUTS) suggestive of benign prostatic hyperplasia (BPH)- focus on the UK. BJU Int. 2015; 115: 508-519.

3. Emberton M, Cornel EB, Bassi PF, Fourcade RO, Gómez JMF, Castro R. Benign prostatic hyperplasia as a progressive disease: a guide to the risk factors and options for medical management. Int J Clin Pract. 2008; 62: 1076-1086.

4. Jacobsen SJ, Jacobson DJ, Girman CJ, et al. Natural History of Prostatism: Risk Factors for Acute Urinary Retention. J Urol. 1996; 155: 595-600

5. Jacobsen SJ, Jacobson DJ, Girman CJ, et al. Treatment for benign prostatic hyperplasia among community dwelling men: the Olmsted County study of urinary symptoms and health status. J Urol. 1999; 162: 1301-1306.

6. Roehrborn CG, McConnell J, Bonilla J, et al. Serum prostate specific antigen is a strong predictor of future prostate growth in men with benign prostatic hyperplasia. PROSCAR long-term efficacy and safety study. J Urol. 2000; 163: 13-20.

7. Simon RM, Howard LE, Moreira DM, et al. Does Prostate Size Predict the Development of Incident Lower Urinary Tract Symptoms in Men with Mild to No Current Symptoms? Results from the REDUCE Trial. Eur Urol. 2016; 69: 885-891.

8. Oelke M, Bachmann A, Descazeaud A; European Association of Urology. EAU guidelines on the treatment and follow-up of non-neurogenic male lower urinary tract symptoms including benign prostatic obstruction. Eur Urol. 2013; 64: 118-140.

9. McConnell JD, Bruskewitz R, Walsh P, et al. The effect of finasteride on the risk of acute urinary retention and the need for surgical treatment among men with benign prostatic hyperplasia. Finasteride Long-Term Efficacy and Safety Study Group. N Engl J Med. 1998; 338: 557-563.

10. Fusco F, Palmieri A, Ficarra V, et al. a1-Blockers Improve Benign Prostatic
Obstruction in Men with Lower Urinary Tract Symptoms: A Systematic Review and Meta-analysis of Urodynamic Studies. Eur Urol. 2016; 69: 1091-1101.

11. McConnell JD, Roehrborn CG, Bautista OM, et al. The long-term effect of doxazosin, finasteride, and combination therapy on the clinical progression of benign prostatic hyperplasia. N Engl J Med. 2003; 349: 2387-2398.

12. Roehrborn CG, Siami P, Barkin J, et al. on behalf of the CombAT Study Group. The Effects of Combination Therapy with Dutasteride and Tamsulosin on Clinical Outcomes in Men with Symptomatic Benign Prostatic Hyperplasia: 4-Year Results from the CombAT Study. Eur Urol. 2010; 57: 123-131.

13. Kolontarev K, Govorov A, Kasyan G, Priymak D, Pushkar D. Current drug therapy of patients with BPH-LUTS with the special emphasis on PDE5 inhibitors. Cent European J Urol. 2016; 69: 398-403.

14. Gupta NK, McVary KT. Re: Risk of Erectile Dysfunction Associated with Use of $5 \alpha$-Reductase Inhibitors for Benign Prostatic Hyperplasia or Alopecia: Population Based Studies Using the Clinical Practice Research Datalink. Eur Urol. 2017; 72: 317-318.

15. Chatziralli IP, Sergentanis TN. Risk factors for intraoperative floppy iris syndrome: a meta-analysis. Ophthalmology. 2011; 118: 730-735.

16. Pushkar D, Tarasov S, Putilovskiy M. Mild-to-moderate Benign Prostatic Hyperplasia (BPH) symptoms: Filling the gap. 16-th Meeting of the Association of Academic European Urologists (AAEU). Dresden, Germany. December 14-16; 2017: 51-52.

17. Epstein OI. The phenomenon of release activity and the hypothesis of 'spatial' homeostasis. Usp Fiziol Nauk. 2013; 44: 54-76.

18. Epstein O. The Spatial Homeostasis Hypothesis. Symmetry. 2018; 10: 103.

19. Bernoulli J, Konkol Y, Vuorikoski H, Yatkin E. Effects of afala and antiestrogen ICl 182,780 in the model of hormonedependent prostate inflammation. Bull Exp Biol Med. 2014; 156: 807-809.
20. Savelieva KV, Borovskaya TG, Kheyfets IA, et al. Comparative study of pharmacological activity of Afala on the model of hormone-induced prostatitis in rats. Bull Exp Biol Med. 2007; 144: 699.

21. Belous AS, Pokrovskii MV, Pokrovskaya TG, et al. Correction of endothelial dysfunction with impaza preparation in complex with enalapril and losartan during modeling of NO deficiency. Bull Exp Biol Med. 2009; 148: 511-513.

22. Chu X, Agmo A. Sexual incentive motivation in old male rats: the effects of sildenafil and a compound (Impaza) stimulating endothelial NO synthase. Pharmacol Biochem Behav. 2008; 89: 209-217.

23. Fortier AH, Holaday JW, Liang H, Dey C, et al. Recombinant Prostate-Specific Antigen Inhibits Angiogenesis in Vitro and in Vivo. The Prostate. 2003; 56: 212-219.

24. Lawrence MG., Lai J, Clements JA. Kallikreins on Steroids: Structure, Function, and Hormonal Regulation of Prostate-Specific Antigen and the Extended Kallikrein Locus. Endocrine Reviews. 2010; 31: 407-446.

25. Förstermann U, Sessa WC. Nitric oxide synthases: regulation and function. Eur Heart J. 2012; 33: 829-837.

26. Williams SA, Singh P, Isaacs JT, Denmeade SR. Does PSA Play a Role as a Promoting Agent during the Initiation and/or Progression of Prostate Cancer. The Prostate. 2007; 67: 312-329.

27. Gudkov A.V. Experience of Long-term Afala Treatment in Benign Prostate Hyperplasia. Bull Exp Biol Med. 2009; 148 (Suppl. 1): 57-60.

28. Neĭmark AI, Isaenko VI, lakovets IAV, Simashkevich AV, Aliev RT. Administration of afala preparation in urological practice. Urologiia. 2009; 3: 67-70.

29. Markel'AL, Zhavbert ES., Tarasov SA, et al. Effect of Impaza on Cardiovascular System. Bull Exp Biol Med. 2009; 148: 518.

30. Neimark Al, Aliev RT, Muzalevskaya NI, et al. Use of Impaza in the Treatment of Erectile Dysfunction in Patients with Essential Hypertension and CHD. Bull Exp Biol Med. 2009; 148: 328. 